\title{
Analysis and Control of Starting Current of Three Phase Induction Motor by Fuzzy Logic Controller
}

\author{
MR.HEMANT AMHIA, SHARDA PATWA \\ LECTURER(EE) M.E.(Control System) JEC JABALPUR
}

\begin{abstract}
Variable speed drives are growing and varying. Drives expanse depend on progress in different part of science like power system, microelectronic, control methods, and so on. electrical drive control. With the use of space vector technique to electrical drive control the, efficiency and reliability of drives increase and volume, weight and cost of them decrease. Due to the improved operating characteristics they give to the equipment control, Escalators pumps, elevators and conveyor belts all operate more effectively if they are soft started. In turn, the SVPWM method and FLC method reduces maintenance, conserves energy and plays a significant part in improving plant performance and operating costs.
\end{abstract}

Keywords: Induction Motor, Space vector decomposition, , Fuzzy Logic PWM Inverter, , IGBT inverter

\section{Introduction}

An important factor in industrial progress during the past five decades has been the increasing sophistication of factory automation which has improved productivity manifold. Manufacturing lines typically involve a variety of variable speed motor drives which serve to power conveyor belts, black start power plants, robot arms, overhead cranes, steel process lines, paper mills, and plastic and fiber processing lines to name only a few. Prior to the 1950s all such applications required the use of a DC motor drive since AC motors were not capable of smoothly varying speed since they inherently operated synchronously or nearly synchronously with the frequency of electrical input. To a large extent, these applications are now serviced by what can be called general-purpose AC drives. In general, such AC drives often feature a cost advantage over their DC counterparts and, in addition, offer lower maintenance, smaller motor size, and improved reliability. However, the control flexibility available with these drives is limited and their application is, in the main, restricted to fan, pump, and compressor types of applications where the speed need be regulated only roughly and where transient response and low-speed performance are not critical. More demanding drives used in machine tools, spindles, high-speed elevators, dynamo meters, mine winders rolling mills, glass float lines, and the like have much more sophisticated requirements and must afford the flexibility to allow for regulation of a number of variables, such as speed, position, acceleration, and torque. Such high-performance applications typically require a high speed holding accuracy and fast transient response. Until recently, such drives were almost exclusively the domain of DC motors combined with various configurations of AC-to-DC converters depending upon the application. With suitable control, however, induction motors have been shown to be more than a match for DC drives in high-performance applications. While control of the induction machine is considerably more complicated than its DC motor counterpart, with continual advancement of microelectronics, these control complexities have essentially been overcome. Such that power electronic equipment which is used widely in motor drives is IGBTs.

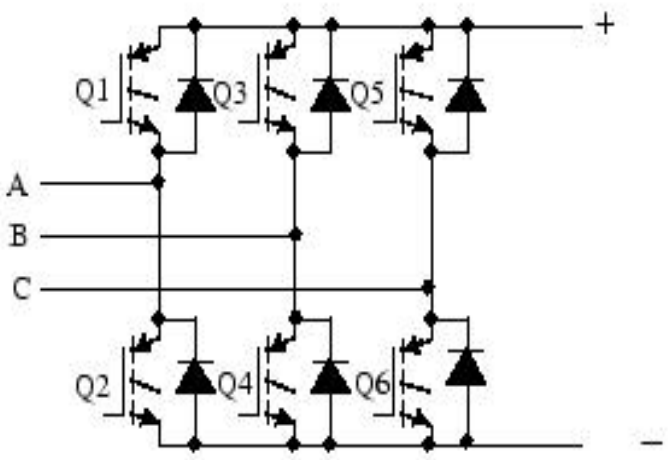

Figure1. IGBT power electronic element 
In the last few decades, the induction motor has evolved from being the constant speed motor to a variable speed, variable torque machine. When application require large amount of power and torque, the induction motor become more efficient to use. With the use of variable voltage, variable frequency (VVVF), the use of induction motor has increased. Variable frequency IGBT inverter fed space vector control method is widely used to control the speed harmonic, and starting current, voltage, rotor speed, output torque of a3 phase squirrel cage induction motor (IM) over a wide range by varying the stator frequency. In particular the IGBT fed space vector control are widely preferred in industries for individual medium to high power variable speed drive system, driving a group of motors connected in parallel at economic cost. The "Insulated Gate Bipolar Transistors (IGBT) is a common choice in modern VFD. The IGBT can switch on and off several thousand times per second and precisely control the power delivered to the motor. The IGBT uses "pulse width modulation" (PWM) technique to simulate a sine wave current at the desired frequency to the motor.

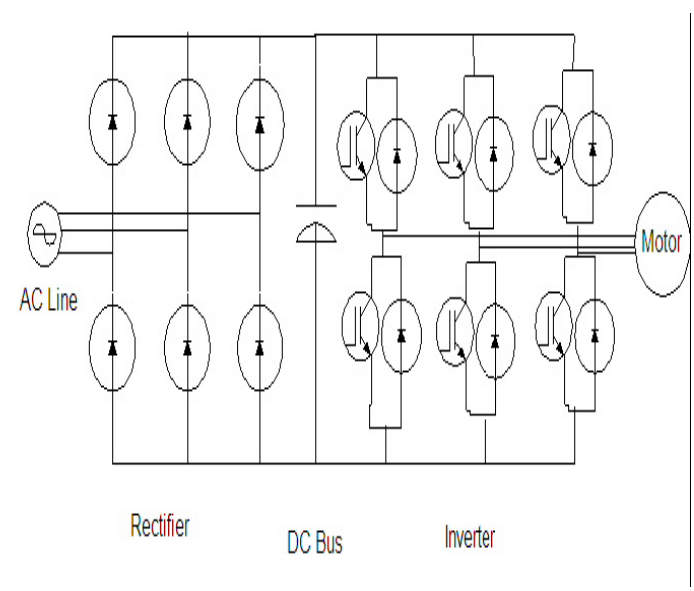

IGBT fed Three Phase Induction Machine

\section{Methodology Of Work}

A simulation model of such induction motor drive system is developed and its dynamic response is verified by observing starting current voltage and torque and control to established acceptability of the model. Here we used transistors based drives. The transistor based drives are capable of both turn on and turn off.

\section{Control Principle (Vector Control Principle)}

AC motors, particularly the squirrel cage induction motor(SCIM), enjoy several inherent advantages like simplicity, reliability, low cost and virtually maintenance free electrical drives. However, for high dynamic performance industrial application, their control remains the challenging problem, because they exhibit significant non-linearity and many of the parameters, mainly the rotor resistance, vary with the operating condition. Field orientation control (FOC) or vector control of an induction motor achieves decoupled torque and flux dynamics leading to independent control of the torque and flux as for a separately excited DC motor. FOC methods are attractive but suffer from one major disadvantage: they are sensitive to motor parameter variations such as the rotor time constant and an incorrect flux measurement or estimation at low speeds Consequently, performance deteriorates and a conventional controller such as a PID is unable to maintain satisfactory performance under these conditions. large majority of variable speed Applications require only speed control in which the torque response is only of secondary interest, more challenging applications such attraction applications, servomotors and the like depend critically upon the ability of the drive to provide a prescribed torque whereupon the speed becomes the variable of secondary interest. The method of torque control in ac machines is called either vector control or, alternatively field orientation. Vector control refers to the manipulation of terminal currents, flux linkages and voltages to affect the motor torque while field orientation refers to the manipulation of the field quantities within the motor itself. Since it is common for machine designers to visualize motor torque production in terms of the air gap flux densities and MMFs instead of currents and fluxes which relate to terminal quantities, it is useful to begin first with a discussion of the relationship between the two viewpoints. The feedback speed control loop generates the active or torque current command iqs*'. The vector rotator receives the torque and excitation current commands Iqs* and ids* from one of the two positions of a switch: the transient position (1) or the steady state position (2). 


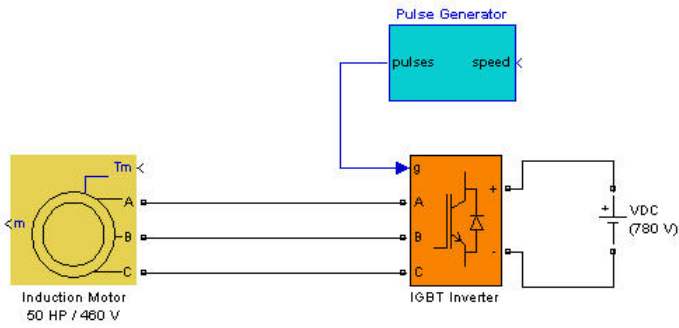

A transistor based induction motor drive

\section{Fuzzy Logic Controller(Flc)}

In the second design approach the basic fuzzy logic controller (FLC), regarded as a kind of variable structure controller (VSC) for which stability and robustness are well established is developed. This follows the interpretation of linguistic IF-THEN rules as a set of controller structures that are switched according to the process states. The mathematical technique called fuzzy logic offers a new approach to improving starting current, voltage, rotor speed and operating torque. Fuzzy logic has evolved from an branch of Mathematics into useful engineering tool. By virtue of its adaptability, it can be applied to problems whose nonlinearity and dynamic nature makes them intractable to solution. Fuzzy logic has been implemented in this development of improved motor control because:

1) Fuzzy logic overcomes the mathematical difficulties of modelling highly non-linear systems.

2) Fuzzy logic responds in a more stable fashion To imprecise readings of feedback control Parameters, such as the dc link current and voltage

3) Fuzzy logic control mathematics and software are simple to develop and flexible for each modification. The fuzzy controller becomes effective at Steady state condition; i.e., when the speed loop error, d_r approaches zero. The voltage perturbation controller is based on changes in input power and stator voltage. Fuzzy logic control has been emphasized for voltage perturbation. Triangular fuzzy sets are used for both inputs and outputs, with a restriction that the output fuzzy sets must be isosceles to simplify defuzzification. Input and output values are represented linguistically.

\section{Tables}

Tables for the fuzzy rule bae

$\mathrm{NB}=$ negative big, $\mathrm{NM}=$ negative medium, $\mathrm{NS}=$ negative small, $\mathrm{ZE}=\mathrm{zero}, \mathrm{PS}=$ positive small, , and $\mathrm{PM}=$ positive medium, $\mathrm{PB}=$ positive big). The rule base table can be read according to the

following example:

IF ERROR (E) is ZERO (Z) AND CHANGE

IN ERROR (CE) is NEGATIVE SMALL (NS),

THEN OUTPUT (DU) is NEGATIVE SMALL (NS).

\begin{tabular}{|l|l|l|l|l|l|l|l|}
\hline $\begin{array}{l}\text { Q } \\
\text { c }\end{array}$ & NB & NM & NS & Z & PS & PM & PB \\
\hline NB & NB & NB & NB & NB & NS & NS & Z \\
\hline $\begin{array}{l}\mathbf{N} \\
\mathbf{M}\end{array}$ & NB & NM & NM & NM & NS & Z & PS \\
\hline NS & NB & NM & NS & NS & Z & PS & PS \\
\hline $\mathbf{Z}$ & NB & NM & NS & Z & PS & PM & PB \\
\hline PS & NS & NS & Z & PS & PS & PM & PB \\
\hline PM & NS & Z & PS & PM & PM & PM & PB \\
\hline PB & Z & PS & PS & PB & PB & PB & PB \\
\hline
\end{tabular}

Speed correction control is needed because the perturbation approach alters motor speed and output power. The motor's output rotor speed should be maintained as constant as possible.

\section{Simulation}

In this paper two case studies have been studied. In Both simulations, it is used simulink and Power sym toolboxes of MATLAB software. In the first case study, a $50 \mathrm{HP}$ induction motor is started and controlled by a PID controller. 3 phase voltages and currents are measured and plotted in the first 3 seconds of its action. Also acceleration curve and output torque are investigated. In the second case, the same motor is started and controlled by a Fuzzy Logic Based controller. The results are shown in figure 10. As it is shown the outputs are 
improved regarding to magnitude of starting currents and also time response of acceleration. For example amplitude of current with a classic PID controller is about 500 A during start-up while with fuzzy logic controller this value reduced to $200 \mathrm{~A}$.

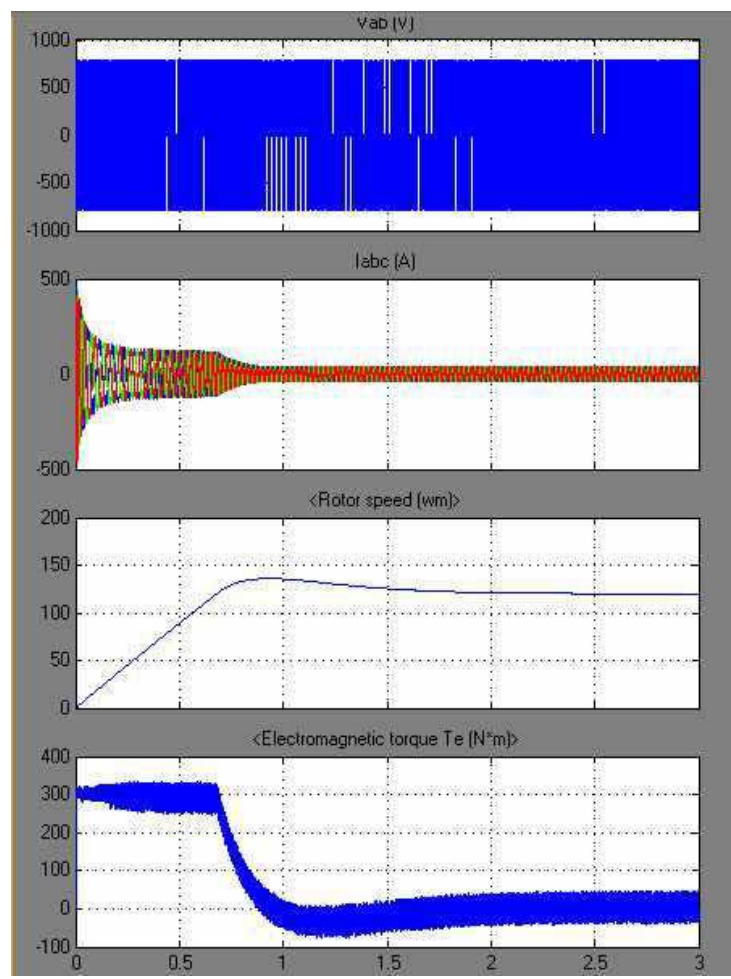

Output Variable of a classic controlled induction Motor from Top to Bottom:Voltage, Current, Rotor Speed, Output Torque

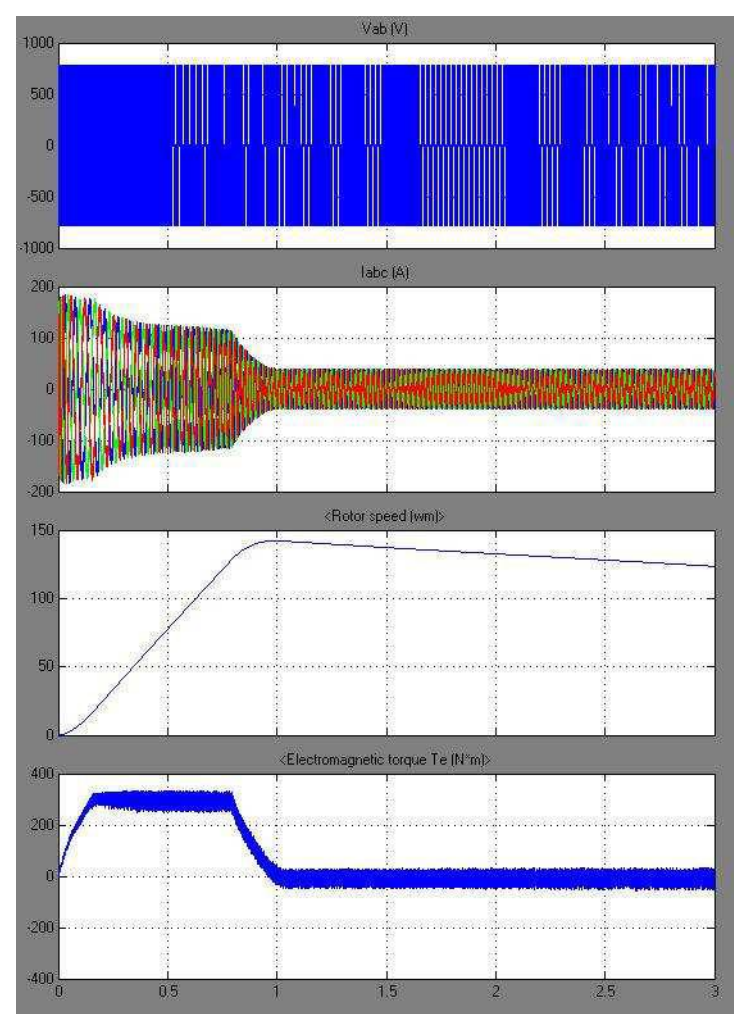

Output Variable of a Fuzzy Logic Controlled induction Motor from Top to Bottom: Voltage, Current, Rotor Speed, Output Torque. 


\section{Conclusion}

An AC induction motor can consume more energy than it actually needs to perform its work, particularly when operated at less than full load conditions. This excess energy is given off by the motor in the form of heat. Idling, cyclic, lightly loaded or oversized motors consume more power than required even when they are not working. With a fuzzy logic controller we can control the amplitude of starting current and also save more energy during this time. In addition the cost and complexity of controller is reduced when it is designed by fuzzy method, because it does not need the exact model of system.

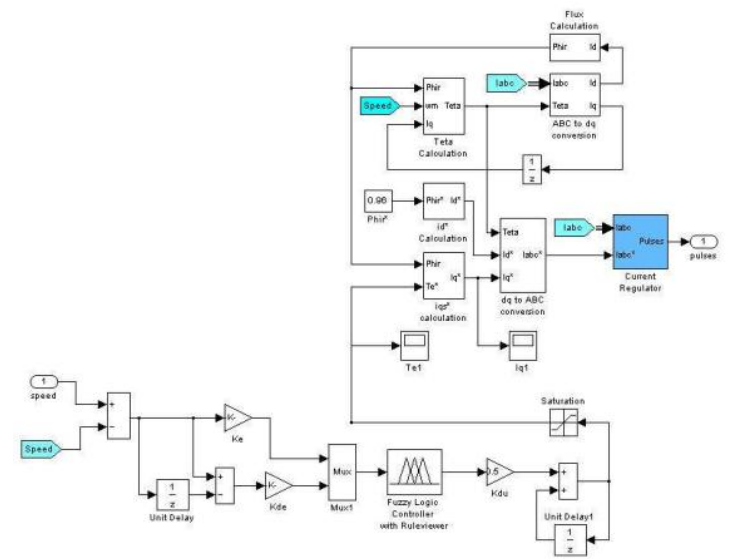

Top: Complete system Bottom: Fuzzy Logic Controller

\section{References}

[1] Bimal Bose, Power Electronics and Motor Drives, Elsevier, Academic Press, 2006

[2] M. AZZEDINE DENAI, S. "AHMED ATTIA, FUZZY AND NEURAL CONTROL OF AN INDUCTION MOTOR" Int. J. Appl. Math. Computer. Sci., 2002, Vol.12.

[3] John G. Cleland and M. Wayne Turner, "Fuzzy Logic Control of Electric Motors and Motor Drives Feasibility Study", United States Air and Energy Engineering Environmental Protection Research Laboratory Agency Research Triangle Park,

[4] J. X. Shin, Z. Q. Zhu, and D. Howe, "Hybrid PI and Fuzzy Logic Speed Control of PM Brushless AC Drives", EPÉE 2001 Graz

[5] Andreas Dannenberg, "Fuzzy Logic Motor Control with MSP430x14x", Texas Instruments, Application Report, SLAA235February 2005.

[6] Y. Milhous, A. Draou, "Performance Analysis of a Fuzzy Logic Based Rotor Resistance Estimator of an Indirect Vector Controlled Induction Motor Drive", Turk J Elce Engine, VL 13, NO. 2, 2005 